ARTICLE

\title{
Orthogonal colloidal quantum dot inks enable efficient multilayer optoelectronic devices
}

Seungjin Lee ${ }^{1,5}$, Min-Jae Choi ${ }^{1,3,5}$, Geetu Sharma², Margherita Biondi ${ }^{1}$, Bin Chen', Se-Woong Baek ${ }^{1,4}$, Amin Morteza Najarian', Maral Vafaie (10 1, Joshua Wicks (10 1, Laxmi Kishore Sagar ${ }^{1}$, Sjoerd Hoogland ${ }^{1}$, F. Pelayo García de Arquer (i) ${ }^{1}$, Oleksandr Voznyy (D) ${ }^{2 \times} \&$ Edward H. Sargent (i) ${ }^{1 凶}$

Surface ligands enable control over the dispersibility of colloidal quantum dots (CQDs) via steric and electrostatic stabilization. Today's device-grade CQD inks have consistently relied on highly polar solvents: this enables facile single-step deposition of multi-hundrednanometer-thick CQD films; but it prevents the realization of CQD film stacks made up of CQDs having different compositions, since polar solvents redisperse underlying films. Here we introduce aromatic ligands to achieve process-orthogonal CQD inks, and enable thereby multifunctional multilayer CQD solids. We explore the effect of the anchoring group of the aromatic ligand on the solubility of CQD inks in weakly-polar solvents, and find that a judicious selection of the anchoring group induces a dipole that provides additional CQDsolvent interactions. This enables colloidal stability without relying on bulky insulating ligands. We showcase the benefit of this ink as the hole transport layer in CQD optoelectronics, achieving an external quantum efficiency of $84 \%$ at $1210 \mathrm{~nm}$.

\footnotetext{
${ }^{1}$ Department of Electrical and Computer Engineering, University of Toronto, 35 St George Street, Toronto, ON M5S 1A4, Canada. ${ }^{2}$ Department of Physical and Environmental Sciences, University of Toronto Scarborough, Scarborough, ON M1C 1A4, Canada. ${ }^{3}$ Present address: Department of Chemical and Biochemical Engineering, Dongguk University, Seoul 04620, Republic of Korea. ${ }^{4}$ Present address: Department of Chemical and Biological Engineering, Korea University, 145 Anam-Ro, Seongbuk-Gu, Seoul 02841, Republic of Korea. ${ }^{5}$ These authors contributed equally: Seungjin Lee, Min-Jae Choi.

凶email: o.voznyy@utoronto.ca; ted.sargent@utoronto.ca
} 
C

olloidal quantum dots (CQDs) have been explored in optoelectronic applications such as photovoltaics (PVs) ${ }^{1-3}$, photodetectors $^{4-6}$, light-emitting $\operatorname{diodes}^{7-9}$, and lasers ${ }^{10,11}$, a testament to their facile bandgap tuning and solution processing. Lead chalcogenide CQDs have the advantage of wideranging bandgap tunability across the visible to the infrared (IR) $(\sim 0.6 \mathrm{eV})$, extending the PV and photodetector response beyond the $\mathrm{c}$-Si bandgap $\left(E_{g}=1.1 \mathrm{eV}\right)$. In light of their IR absorption, CQDs are promising materials for use in short-wavelength IR photodetectors $^{12}$ and as back cells ${ }^{13,14}$ in tandems with $\mathrm{Si}\left(E_{g}=\right.$ $1.1 \mathrm{eV})$ and perovskites $(1.58-1.68 \mathrm{eV})$.

The synthesis of CQDs relies on the use of long insulating ligands such as oleic acid (OA) and oleylamine. For applications in optoelectronic devices, these must be replaced with shorter ligands to improve carrier transport in CQD films. Early device prototypes employed a layer-by-layer (LBL) deposition strategy, known as solid-state ligand exchange, to fabricate CQD films ${ }^{1,15-18}$. However, the LBL solid-state ligand exchange process is incompatible with scalable methods of manufacture such as roll-to-roll. Solid-state ligand exchanges also lead to inhomogeneous energy states in CQD films ${ }^{19-23}$.

Solution-phase ligand exchanges have been developed to overcome these issues ${ }^{24-29}$ : they enable the formation of smooth and crack-free CQD films through a single, $100 \%$-materials-utilization, deposition. High-quality CQD inks based on short ligands, such as lead halides ${ }^{25-27}$, acetate salts ${ }^{26,27}$, and thiols 3,28 , have been demonstrated.

These works have relied on the use of highly polar solvents. This approach stands in the way of the creation of multifunctional multilayer devices-structures in which the different layers incorporate distinct sizes and composition of quantum dots - since highly polar solvents disperse the prior layers. For this reason, today's highest-performing CQD optoelectronic devices rely on a solid-state ligand exchange step ${ }^{1,3,25-27}$ to construct the final CQD hole transport layer (HTL) atop the main active layer.

These considerations mandate the development of orthogonal CQD inks that are sufficiently nonpolar to preserve the underlying layers. However, conventional nonpolar and low-polarity CQD inks have relied on long electrically insulating hydrophobic ligands since shorter ligands produce CQD aggregation in lowpolarity solvents.

We reasoned that-since aromatic rings are known to be highly polarizable-a judicious selection of aromatic ligands, including of their anchoring group, would induce a dipole for increased interaction with a weakly polar solvent, enabling colloidal stability.

Here we report as a result a ligand strategy that enables CQD inks based on weakly polar solvents. Using a combination of experiment and computation, we explore the effect of the anchoring group of the ligand on the solubility of the CQD ink. We find that the stronger electron-withdrawing effect of the carboxyl group results in polarization of the aromatic ring relative to the thiol group, enabling benzoic acid (BA)-exchanged CQDs to be stabilized in chlorobenzene. In contrast, benzenethiol (BT)exchanged CQDs aggregate and precipitate.

We deploy the weakly polar CQD inks to devise smooth and crack-free HTLs that exhibit reduced trap densities compared to conventional ethanedithiol (EDT)-exchanged HTLs reliant on solid-state ligand exchange. Single-step spin-coating with chlorobenzene enables the fabrication of HTLs without degradation of the CQD layer beneath, whereas the solid-state ligand exchange procedure for the deposition of the EDT-exchanged HTL leads to trap states in the underlying light-absorbing layer. We realize fully ink-based CQD PV, achieving an external quantum efficiency (EQE) of $84 \%$ at $1210 \mathrm{~nm}$. This results in addition of
$1.43 \%$ and $3.57 \%$ additional solar-power points atop those of silicon and perovskite solar cells, respectively.

\section{Results}

Synthesis of weakly polar CQD inks. Conventional nonpolar CQD inks rely on bulky ligands, leading to compromised charge transport. In a previous report, 4-methylbenzenethiol $\left(4-\mathrm{CH}_{3}-\right.$ $\mathrm{BT}$ )-exchanged $\mathrm{PbS}$ CQDs were stabilized in weakly polar solvents such as dichloromethane and dichlorobenzene ${ }^{19}$. However, the 4- $\mathrm{CH}_{3}$-BT-exchanged CQD films showed limited charge transport, resulting in low $\mathrm{PV}$ performance and a power conversion efficiency (PCE) of $1.34 \%$. We sought therefore to use a shorter BT ligand to synthesize a weakly polar CQD ink without the methyl pending group required in previous works; however, the BT-exchanged PbS CQDs were substantially insoluble in weakly polar solvents including chloroform, dichloromethane, and chlorobenzene (Supplementary Fig. 1a).

Colloidal stabilization of CQDs requires a sufficient CQDsolvent interaction to overcome the CQD-CQD attraction. Given that aromatic molecules are highly polarizable, we reasoned that the anchoring group of ligands enables control over the polarization of a benzene ring, providing an additional means to enhance CQD-solvent interactions in the case when the solvent is weakly polar. We reasoned that the carboxyl group induce a stronger dipolar polarization of the aromatic ring compared to the thiol group ${ }^{30}$, and that this can enhance interactions of the CQD with a weakly polar solvent. For this reason, we sought to use BA ligands to synthesize CQD inks stabilized in weakly polar solvents (Fig. 1a) toward the ultimate goal of efficient charge transport in final CQD films. This allowed colloids in a variety of weakly polar solvents (Supplementary Fig. 1b) that included chlorobenzene, toluene, dichloromethane, chloroform, and anisole, without the need for an additional pending methyl group previously employed.

We carried out density functional theory (DFT) calculations to elucidate the effect of the ligand anchoring group and the pendant methyl group on the solubility of CQDs. We studied a series of four different aromatic ligands and calculated the interaction energies of exchanged CQDs with chlorobenzene solvent (Fig. 1b and Supplementary Table 1). The presence of a methyl group in the para position enhanced the interaction energy for both BTand BA-functionalized CQDs, supporting previous findings that methyl group improves the solubility of CQDs in weakly polar solvents. Experimental results showed that without a methyl group, BT-exchanged CQDs are insoluble in chlorobenzene.

The BA-exchanged CQDs showed significantly higher interaction energy with chlorobenzene despite the fact that the outer surface of the ligand is identical-a finding consistent with the hypothesized polarization effect. The computed electrostatic potential of an entire CQD capped with ligands reveals larger pockets of positive potential. These pockets are available for interaction with the solvent dipole (Fig. 1c), and are consistent with the electrostatic potentials of isolated ligands (Fig. 1d). Experimentally, we found that-despite the improvement of solubility in the presence of one methyl group-4- $\mathrm{CH}_{3}-\mathrm{BT}$ exchanged CQDs showed lower solubility than the BA-exchanged CQDs. The BA-exchanged CQDs remained stable as colloids, producing no observable aggregation following 6 months' storage in air (Supplementary Fig. 2).

To challenge the versatility of the ligand exchange strategy, we sought to deploy it also on InAs and InP CQDs. We used different combinations of solvents/solvent mixtures depending on the CQDs since the dynamics of ligand exchange depend on the CQD surface chemistry. With optimal ligand exchange 
a

4-Methylbenzenethiol 4-Methylbenzoic acid (4- $\left.\mathrm{CH}_{3}-\mathrm{BT}\right)$

$\left(4-\mathrm{CH}_{3}-\mathrm{BA}\right)$

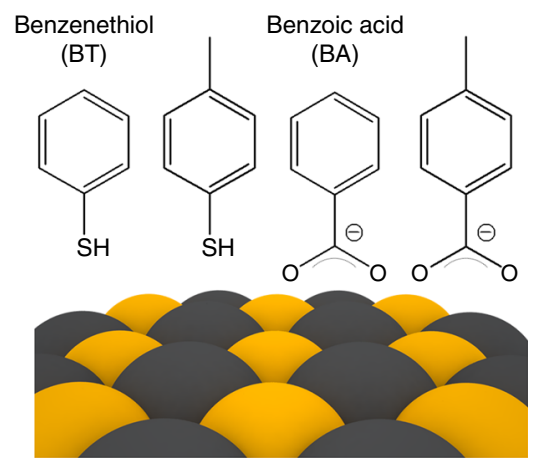

e

BA-exchanged CQDs

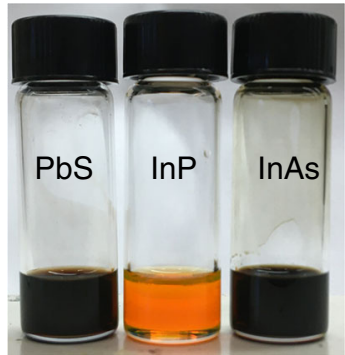

b

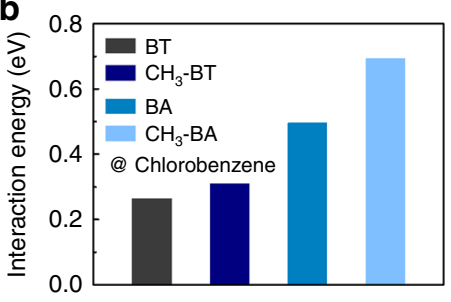

c

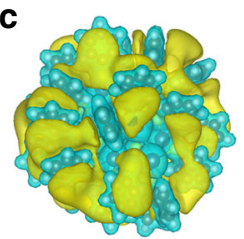

BT-exchanged PbS

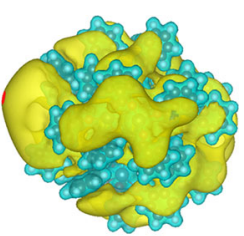

d

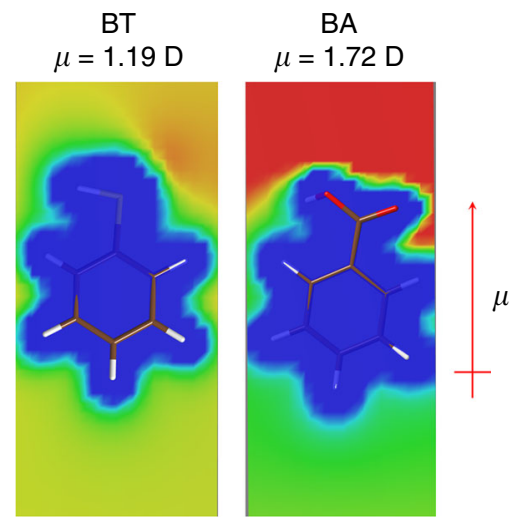

f

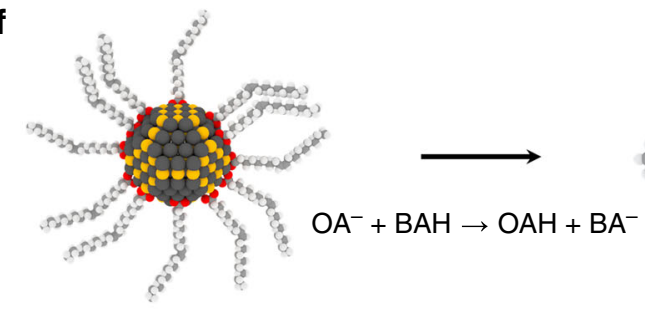

BA-exchanged PbS

.

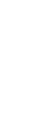


a

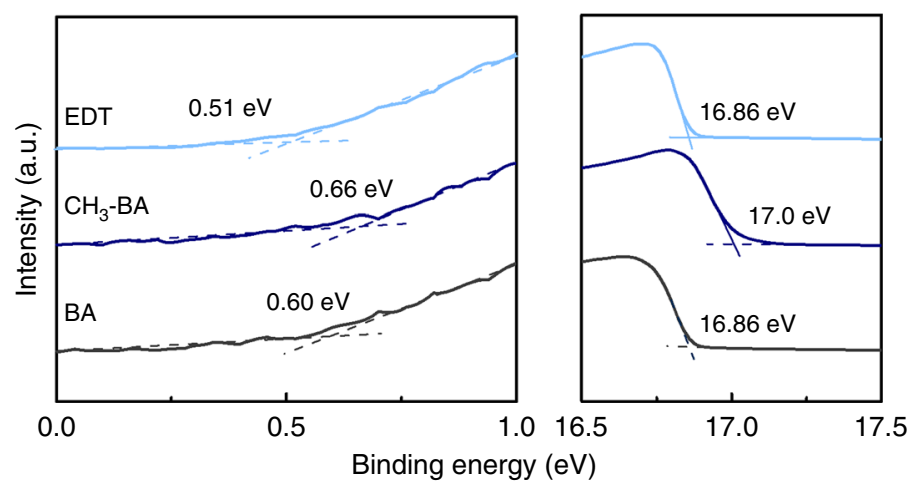

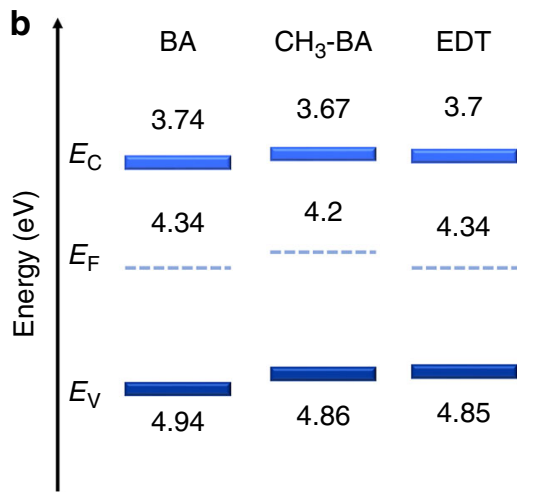
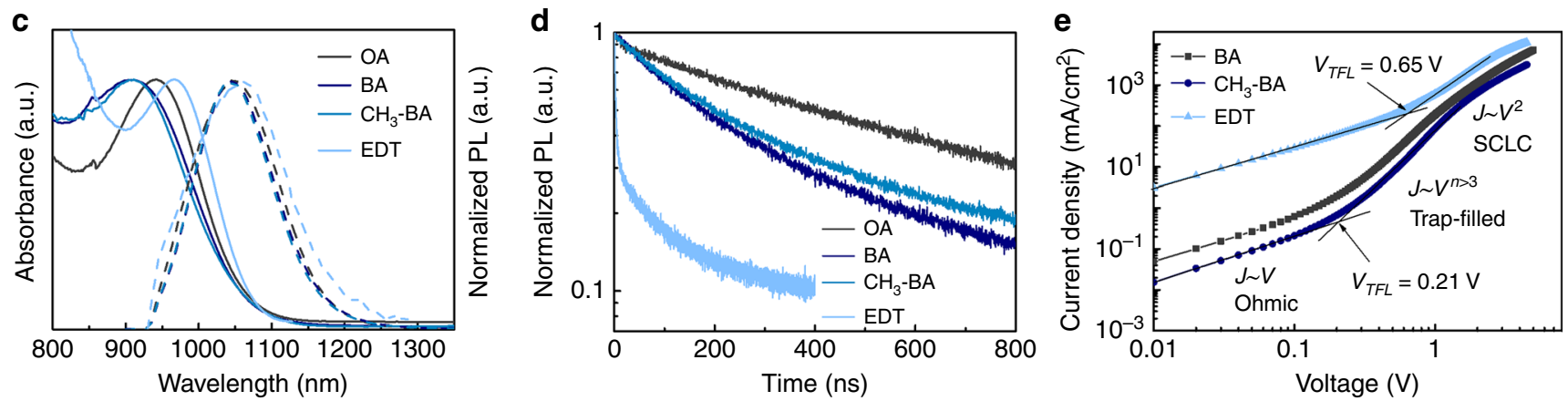

Fig. 2 Characterization of HTLs. a UPS spectra of the BA-functionalized CQD films and the conventional EDT-exchanged film. $\mathbf{b}$ Energy levels of the BAfunctionalized CQD films and the EDT-exchanged film. c Normalized absorption and PL spectra of CQD films before and after ligand exchange. $\mathbf{d}$ Timeresolved PL spectra of CQD films before and after ligand exchange. e J-V characteristics of HODs with different HTLs.

decay time. Using an independent method-space-charge-limited current trap-filling studies of Fig. $2 \mathrm{e}$-we found that the EDTexchanged CQD films showed a $3.5 \times$ higher trap state density than did the BA-functionalized CQD films. We therefore offer that a significant contributor to the faster PL decay time in the EDT-exchanged CQD films can include the effect of trap states.

To investigate further the mobility and trap density of CQDs exchanged using $\mathrm{BA}, 4-\mathrm{CH}_{3}-\mathrm{BA}$, and EDT, we measured dark currents of hole-only devices (HODs) with the structure of ITO/ $\mathrm{NiO}_{x} / \mathrm{PbS} \mathrm{CQDs} / \mathrm{MoO}_{3} / \mathrm{Au}$ (Fig. 2e). The trap density is estimated from the trap-filled regime, while the hole mobility is estimated from the space charge-limited current (SCLC) regime (Supplementary Note 1). From the SCLC regime, the hole mobilities were estimated to be $1 \times 10^{-4}, 7 \times 10^{-5}$, and $2 \times 10^{-4}$ $\mathrm{cm}^{-2} \mathrm{~V}^{-1} \mathrm{~s}^{-1}$ for BA-, 4-CH $\mathrm{CH}_{3}-\mathrm{BA}-$, and EDT-exchanged CQD films, respectively (Supplementary Table 3 ). As the ligand size decreases, the hole mobility of the CQD films increases, a finding we assign to the enhanced inter-dot coupling. From the trap-filled regime, we estimated the trap densities to be $5 \times 10^{16}, 5 \times 10^{16}$, and $2 \times 10^{17} \mathrm{~cm}^{-3}$ for the CQD films with $\mathrm{BA}, 4-\mathrm{CH}_{3}-\mathrm{BA}$, and EDT, respectively. The BA-functionalized HTLs fabricated with the CQD inks thus showed lower trap densities compared to the EDT-exchanged HTL fabricated via solid-state ligand exchange.

To compare the morphologies of the CQD films exchanged with $\mathrm{BA}, 4-\mathrm{CH}_{3}-\mathrm{BA}$ and $\mathrm{EDT}$, we carried out scanning electron microscopy (SEM) and atomic force microscopy (AFM) studies (Supplementary Fig. 4). The BA-functionalized CQD films fabricated using CQD inks exhibited continuous and smooth morphologies without cracks and pinholes. A root mean square roughness $<1 \mathrm{~nm}$ was achieved for the BA-functionalized CQD films. In contrast, the EDT-exchanged CQD film showed a rough morphology with cracks. These cracks have previously been noted for CQD films fabricated via solid-state ligand exchange, a result of the considerable volume contraction that follows the exchange of the bulky OA ligands with small ligands ${ }^{19-21}$. The rough EDTexchanged film may contribute to increased leakage currents in devices.

Orthogonality between polar and weakly polar CQD inks. To fabricate the light-absorbing layer, we used halidepassivated CQDs dispersed in butylamine:dimethylformamide $(\mathrm{DMF})(4: 1 \mathrm{v} / \mathrm{v})$. The use of an orthogonal solvent (chlorobenzene) to fabricate BA-functionalized HTLs purposes to prevent degradation of the underlying light-absorbing layer. Solid-state EDT-exchanged CQD films are obtained by deposition of OA-capped CQDs, soaking the OA-capped film in EDT/ acetonitrile (ACN) solution, and washing away unbound ligands by spin-coating ACN. These procedures result in partial loss of ligands in the underlying light-absorbing layer ${ }^{22,23,33}$.

To investigate the effect of the protocols for depositing HTLs on the underlying CQD layer, we measured absorption and PL spectra of the light-absorbing layers after spin-coating chlorobenzene (for BA-functionalized films) and soaking with ACN (for EDT film) (Fig. 3a). After we soaked using ACN, CQD films exhibited a red-shifted and broader PL spectrum, as well as a considerably weaker PL intensity compared to pristine CQD films (Fig. 3a and Supplementary Fig. 5), indicating the creation of defect states in the underlying CQDs. In contrast, the chlorobenzene-treated CQD films showed a similar PL intensity and spectrum compared to the pristine film, showing no remarkable change in optical and photophysical characteristics of the underlying CQDs.

The creation of localized trap states was investigated by observing the bandtail absorption of CQD films before and after solvent treatments (Fig. 3b) ${ }^{26,34}$. The chlorobenzene-treated CQD films showed unchanged bandtail absorption, whereas bandtail absorption was observed for the ACN-soaked film. To understand 
a
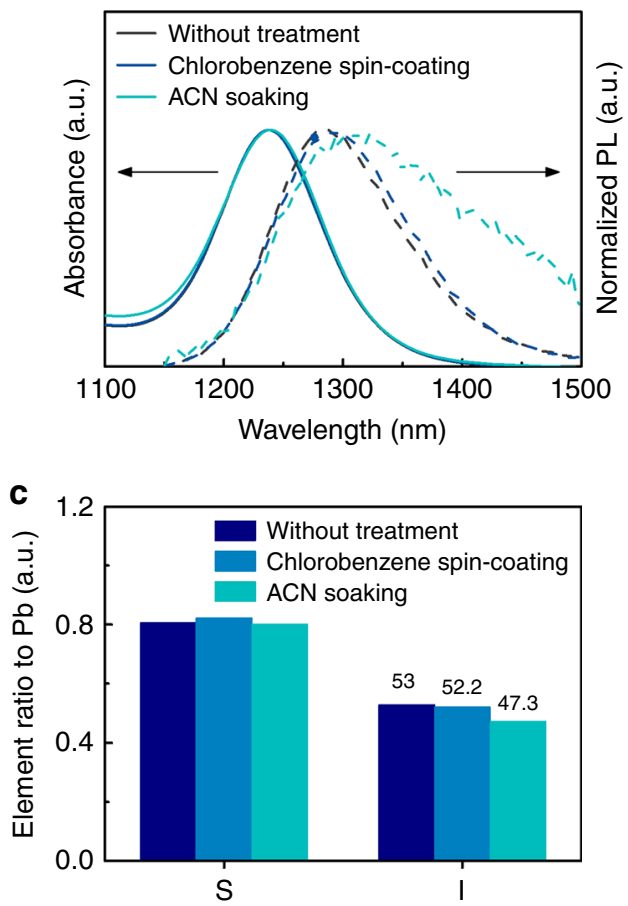

b

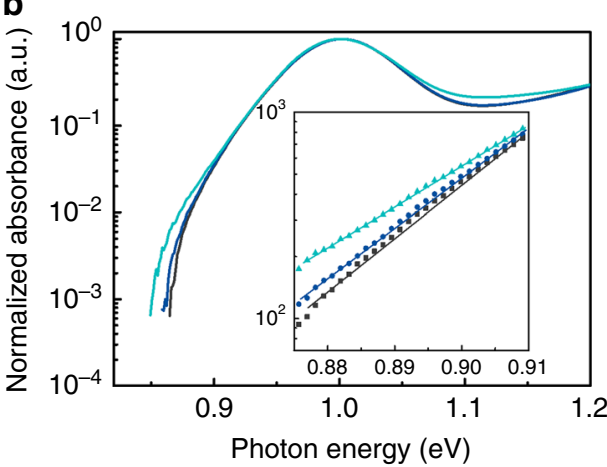

d

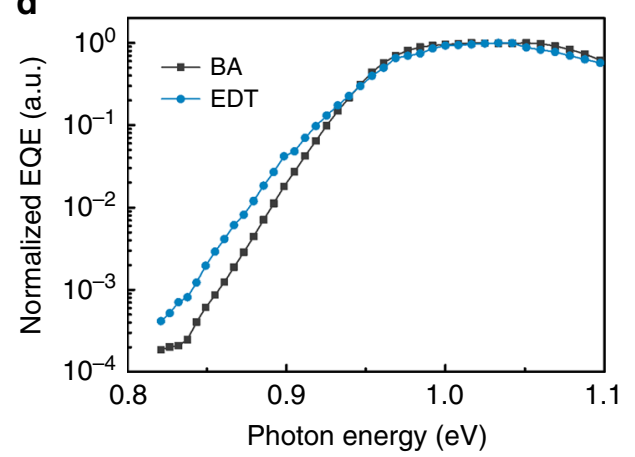

Fig. 3 Degradation of light-absorbing layer upon the application of HTL-deposition protocols. a Normalized absorption and PL spectra of CQD films before and after solvent treatments. b Logarithmic-scale absorption spectra of CQD films to investigate bandtail absorption. $\mathbf{c}$ Element ratio-to-lead of CQD films before and after solvent treatments. d HDR EQE measurement of devices with BA HTL and EDT HTL to investigate the carrier distribution over the energy range below the bandgap.

the origins of trap states in the CQD film following soaking with $\mathrm{ACN}$, we performed X-ray photoelectron spectroscopy (XPS) to study the elemental compositions of CQD films. These revealed that the variation in the iodide to $\mathrm{Pb}$ ratio is negligible after spincoating chlorobenzene, while the iodide ratio to $\mathrm{Pb}$ was reduced from 53 to $47 \%$ after soaking with ACN. We conclude that a portion of iodide ligands detached and washed away from the QD surface during soaking with ACN, leaving the CQD surface underpassivated. This we correlate with trap states arising due to incomplete passivation of the CQD surface upon the partial loss of iodide ligands.

To investigate energetic disorder in devices, we measured high dynamic range EQE (HDR EQE) for devices with the architecture of ITO/ZnO/IR-absorbing PbS CQD layer $\left(E_{g}=1.0 \mathrm{eV}\right) / \mathrm{HTLs}$ $\left(E_{g}=1.3 \mathrm{eV}\right) / \mathrm{Au}$ (Fig. $\left.3 \mathrm{~d}\right)$. This technique reports on the density of states in bandtail states ${ }^{26,34}$. Devices fabricated with BA HTL showed a steeper slope of the bandtail EQE than devices fabricated with EDT HTL.

PV performance. To test the performance of CQD PV devices employing different HTLs, we measured the EQE spectra and the current density-voltage $(J-V)$ characteristics of PbS CQD PVs fabricated with $\mathrm{BA}, 4-\mathrm{CH}_{3}-\mathrm{BA}$, and EDT. We studied these under AM 1.5 illumination passed through a $1100-n m$ cutoff silicon filter (Fig. 4a, b). The CQD PVs with orthogonal CQD inks exhibited an EQE of $84 \%$ at the excitonic peak $(1210 \mathrm{~nm})$ and also a higher open-circuit voltage $\left(V_{\mathrm{OC}}\right)$ than devices with EDT. A slightly lower fill factor (FF) was observed for devices with $\mathrm{CH}_{3}$-BA due to the lower hole mobility of the $\mathrm{CH}_{3}$-BA-excahnged layer. Si-filtered PCE values (the 1100 -nm cutoff silicon filter) from more than 60 devices ( 20 devices for each ligand type) reveal that BA-based devices led to a statistically significant increase in
PCE compared to devices with $\mathrm{CH}_{3}-\mathrm{BA}$ and EDT as seen in Fig. 4c. Specifically, the Si-filtered PCE was $1.4 \pm 0.02 \%$ for BA devices compared to $1.28 \pm 0.02 \%$ for $\mathrm{CH} 3-\mathrm{BA}$ and $1.28 \pm 0.02 \%$ for EDT. The best PV performance with a record PCE of $1.43 \%$, a $V_{\mathrm{OC}}$ of $0.438 \mathrm{~V}$, a short-circuit current density $\left(J_{\mathrm{SC}}\right)$ of $5.34 \mathrm{~mA}$ $\mathrm{cm}^{-2}$, and a FF of $61.3 \%$ was achieved for the BA-based device with the 1100-nm cutoff silicon filter (Supplementary Table 4). We then investigated the operating stability of unencapsulated devices fabricated with the BA HTL and the EDT HTL, investigating at the maximum power point (MPP) under AM 1.5G illumination and under nitrogen flow (Fig. 4d). BA-based devices showed considerably improved operational stability at MPP compared to EDT-based devices, retaining over $80 \%$ of initial PCE following continuous operation for $60 \mathrm{~h}$ under 1-sun illumination.

We then characterized the performance of $4 \mathrm{~T}$ tandem cells by stacking BA-based CQD PV with a semitransparent perovskite front cell. The semitransparent perovskite front cell was prepared with the structure $\mathrm{ITO} / \mathrm{SnO}_{2} /$ perovskite $(1.63 \mathrm{eV}) /$ spiro- $\mathrm{MeO}$ $\mathrm{TAD} / \mathrm{MoO}_{3} /$ indium zinc oxide $/ \mathrm{MgF}_{2}$. The semitransparent perovskite solar cell showed a reverse scan $J_{\mathrm{SC}}$ of $22.1 \mathrm{~mA} \mathrm{~cm}^{-2}$ and PCE of $18.5 \%$ (Supplementary Fig. 6). Figure $4 \mathrm{~d}$, f combines the $4 \mathrm{~T} J-V$ characteristics and EQE spectra of each constituent cell in the $4 \mathrm{~T}$ structure. BA-based CQD PV devices provide an additional $J_{S C}$ of $12.6 \mathrm{~mA} \mathrm{~cm}^{-2}$ and PCE of $3.57 \%$ on top of the semitransparent perovskite solar cell. The PV parameters are summarized in Supplementary Table 4. The all-solutionprocessed perovskite/CQD tandems show excellent photonharvesting capability, achieving a $J_{\mathrm{SC}}$ of $34.7 \mathrm{~mA} \mathrm{~cm}^{-2}$ and a PCE of $22 \%$.

We then tested the performance of PbS CQD photodetectors, comparing the use of the BA HTL and EDT HTL with respect to dark $J-V$ curve, specific detectivity $\left(D^{*}\right)$, and transient 

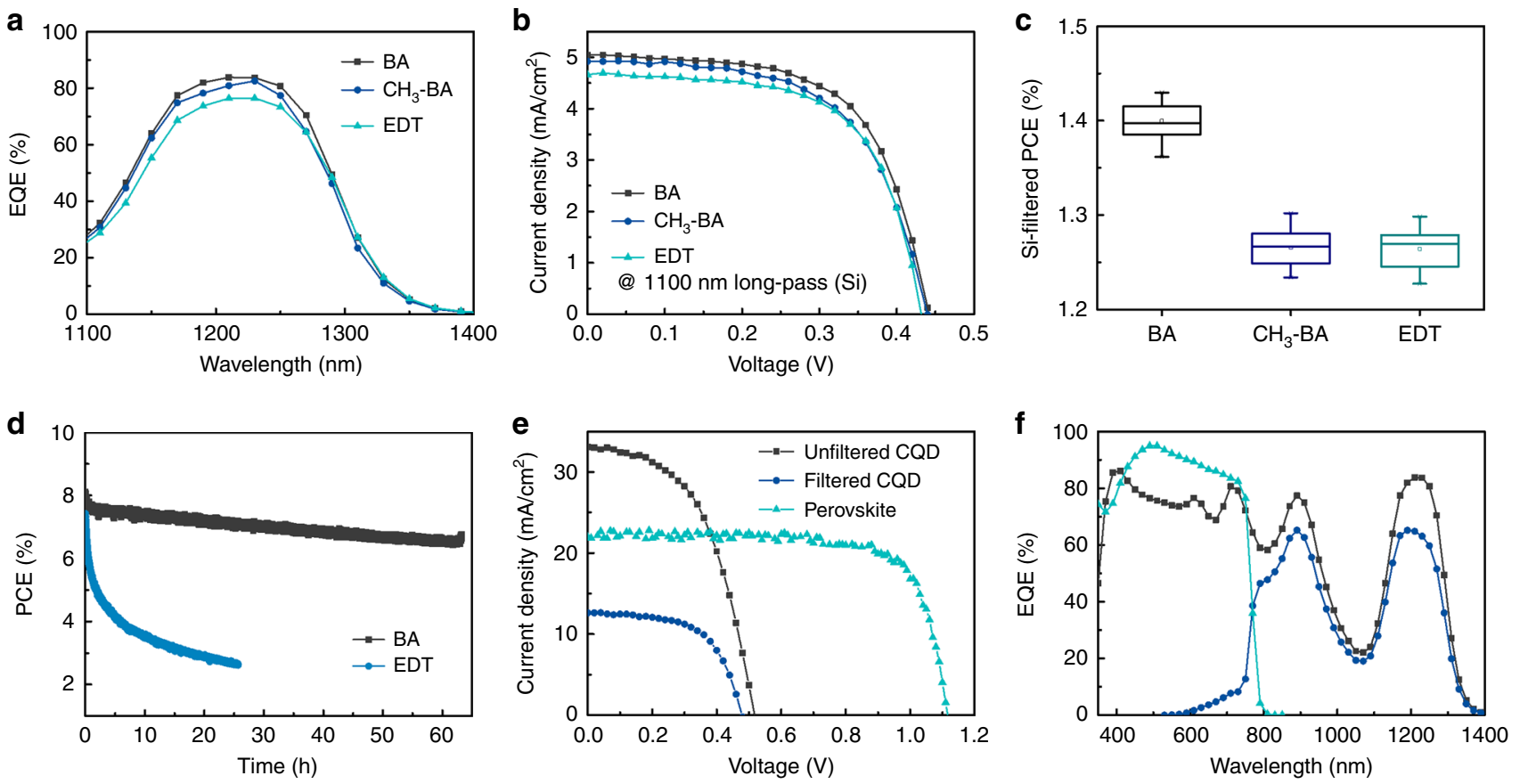

Fig. 4 CQD PV performance. a J-V characteristics of CQD PVs with different HTLs under AM 1.5 illumination with 1100-nm cutoff silicon filter. b EQE spectra of CQD PVs with different HTLs beyond $1100 \mathrm{~nm}$. c Statistics of device performance with different HTLs using 1100-nm cutoff silicon filter. d MPP stability of unencapsulated CQD PVs with BA HTL and EDT HTL under continuous 1-sun illumination and nitrogen flow. e J-V characteristics and $\mathbf{f}$ EQE spectra of perovskite front cell and CQD back cell with BA HTL in the 4T tandem configuration.

photocurrent (TPC) response (Supplementary Note 2 and Supplementary Fig. 7). The BA-based photodetectors showed lower dark current density and noise equivalent power compared to EDT-based photodetectors (Supplementary Fig. 7a). To characterize sensitivity, we measured $D^{*}$ for the photodetectors with BA and EDT (Supplementary Fig. 7b). The BA-based photodetectors showed a higher peak $D^{*}$ value of $1.4 \times 10^{12}$ Jones at $1230 \mathrm{~nm}$ than the EDT-based photodetector $\left(1.0 \times 10^{12}\right.$ Jones $)$. The high $D^{*}$ of the BA-based photodetector was achieved through the combination of high EQE and low noise.

\section{Discussion}

In summary, we investigated the effect of ligand functional groups on the solubility of CQDs in weakly polar solvents. We employed aromatic ligands and embarked on a combination of experimental and computational studies. These revealed that the ligand dipole is a critical parameter for predicting the ink solubility. BA-functionalized CQDs are more soluble in chlorobenzene than BT-functionalized CQDs, a finding we assign to the stronger electrostatic potential of the BA ligand bound to the CQD surface. The development of weakly polar CQD inks enables smooth and crack-free HTL films that exhibit reduced trap density compared to conventional HTLs fabricated using solid-state ligand exchange. The chemical orthogonality of weakly polar CQD inks enables the fabrication of HTLs without degradation of the underlying light-absorbing layer, whereas the solidstate ligand exchange gives rise to trap states in the layer beneath. As a result, we fabricate the solid-state ligand exchange-free CQD PVs, achieving an EQE of $84 \%$ at $1210 \mathrm{~nm}$. This results in addition of $1.43 \%$ and $3.57 \%$ extra efficiency on top of silicon and perovskite solar cells, respectively.

\section{Methods}

Materials. BA (99.5\%), 4- $\mathrm{CH}_{3}-\mathrm{BA}(98 \%), \mathrm{BT}(97 \%), 4-\mathrm{CH}_{3}-\mathrm{BT}(98 \%)$, chlorobenzene ( $99.8 \%$, anhydrous), butylamine (99.5\%), DMF ( $99.8 \%$, anhydrous), and hexane $(99 \%)$ were purchased from Sigma-Aldrich and used without further purification. Lead iodide beads, lead bromide, and sodium acetate powders were purchased from Alfa Aesar.

Synthesis of weakly polar CQD inks for HTLs. OA-capped CQDs were synthesized using a previously published method ${ }^{35}$. Orthogonal CQD inks for HTLs were prepared in a nitrogen-filled glovebox. The ligand solution was prepared by dissolving the functionalized aromatic ligands $(0.3 \mathrm{M})$ in an appropriate solvent/ solvent mixture (Supplementary Table 2). For BT-functionalized ligands, the same molar amount of triethylamine (TEA) was added in the ligand solutions to deprotonate BT-functionalized ligands. The addition of TEA improves the solubility of 4- $\mathrm{CH}_{3}-\mathrm{BT}$-exchanged CQDs in chlorobenzene (Supplementary Fig. 8). In contrast, the BA ligands are readily deprotonated without the addition of TEA, a result of the stronger acidity than the OA. As a result, we did not observe a noticeable difference in the solubility of BA-functionalized CQDs upon the addition of TEA. One milliliter of the ligand solution was added dropwise to $1 \mathrm{~mL}$ of CQD solution ( $20 \mathrm{mg} \mathrm{mL}^{-1}$ in toluene) with stirring, and the solution was stirred for $10 \mathrm{~min} .2 .5 \mathrm{~mL}$ of hexane was added to precipitate the exchanged CQDs and then centrifuged at $7000 \mathrm{rpm}$ for $2 \mathrm{~min}$. For the purification of CQDs, the precipitated CQDs were dispersed in $1 \mathrm{~mL}$ of chlorobenzene and then $1.5 \mathrm{~mL}$ of hexane was added to precipitate the CQDs. To completely remove the OA byproduct and the residual ligands, the exchanged CQDs were washed with the purification steps three times.

Synthesis of polar CQD inks for light-absorbing layers. The preparation of CQD inks for light-absorbing layer was carried out in air. The ligand solution was prepared by dissolving lead iodide $(0.1 \mathrm{M})$, lead bromide $(0.02 \mathrm{M})$, and sodium acetate $(0.04 \mathrm{M})$ in DMF. A $5 \mathrm{~mL}$ of CQD solution dissolved in octane $\left(6 \mathrm{mg} \mathrm{mL}^{-1}\right)$ was added to $5 \mathrm{~mL}$ of ligand solution. After vigorously vortexing for $5 \mathrm{~min}$, CQDs were transferred to DMF phase, and the DMF solution was washed with octane three times to remove residual OA ligands. The CQD solution was precipitated by adding toluene and dried in a vacuum. The CQDs were dispersed in butylamine:DMF (4:1 v/v).

CQD PV fabrication. All fabrication processes were carried out in the air. The $\mathrm{ZnO}$ nanoparticles were synthesized using a published method $^{10}$. The $\mathrm{ZnO}$ solution was spin-cast onto ITO substrate at $4000 \mathrm{rpm}$ for $25 \mathrm{~s}$. Then, the halide-passivated PbS CQDs as light-absorbing layer were deposited onto the $\mathrm{ZnO} / \mathrm{ITO}$ substrate by dropping the CQD ink $\left(300 \mathrm{mg} \mathrm{mL}^{-1}\right)$ while the substrate is spinning at $800 \mathrm{rpm}$. Then, orthogonal CQD inks $\left(20 \mathrm{mg} \mathrm{mL}^{-1}\right)$ were spin-cast to fabricate HTLs at $2500 \mathrm{rpm}$ for $25 \mathrm{~s}$. For the EDT-exchanged HTL, OA-capped CQDs $\left(25 \mathrm{mg} \mathrm{mL}^{-1}\right)$ were spin-cast at $2500 \mathrm{rpm}$ for $10 \mathrm{~s}$, and soaked with a 0.01-vol.\% EDT solution in ACN for $30 \mathrm{~s}$, and then followed by three times of washing with ACN. Two EDTexchanged layers were deposited to avoid direct contact between the lightabsorbing layer and the top electrode due to numerous cracks in the EDT- 
exchanged layer. Finally, a 120-nm Au electrode was deposited by e-beam evaporation.

CQD PV characterization. The active area $\left(0.049 \mathrm{~cm}^{2}\right)$ was determined by placing an aperture between the devices and the AM 1.5 solar simulator (Sciencetech class A). The $J-V$ characteristics were measured using a Keithley 2400 source meter under simulated AM 1.5 illumination and a nitrogen atmosphere. The light intensity was calibrated using a reference solar cell (Newport). The $J-V$ curves of CQD PVs were scanned from -0.70 to $+0.1 \mathrm{~V}$ at $0.02-\mathrm{V}$ interval steps without wait time between voltage steps. During the CQD:perovskite 4T measurement, CQD devices were masked by $0.049 \mathrm{~cm}^{2}$ of aperture and placed behind the semitransparent perovskite front cell.

A monochromatic illumination (400-W Xe lamp) with appropriate cutoff filters was used as an excitation source. For EQE calibration, Newport 818-UV and Newport 838-IR photodetectors were used. The response was measured at short-circuit conditions with a Lakeshore preamplifier feeding into a Stanford Research 830 lock-in amplifier. HDR EQE was measured at the sensitivity of the preamplifier of $1 \mathrm{nA} \mathrm{V}^{-1}$, ensuring the sufficient resolution of the EQEs at the IR region $>1100 \mathrm{~nm}$.

CQD photodetector characterization. The average noise current was measured using multiple readings from a SR830 lock-in using its noise measurement system. The detector was connected with a low-noise SR570 preamplifier to the lock-in. The noise currents of photodetectors were measured at a frequency of $1 \mathrm{kHz}$ under zero bias. For TPC measurement, femtosecond laser pulses $(1030 \mathrm{~nm}, 5-\mathrm{kHz}$ repetition rate) generated by a Yb:KGW laser (Pharos, Light Conversion), passed through an optical parametric amplifier (Orpheus, Light Conversion) selected for 600 -nm wavelength. The signals were recorded using an oscilloscope with an input impedance of $50 \Omega$.

DFT calculations. DFT calculations were performed using the Quickstep module of the CP2K software ${ }^{36}$. Goedecker-Teter-Hutter pseudopotentials ${ }^{37}$ within the generalized gradient approximation with Perdew-Burker-Ernzerhoff exchangecorrelation functional ${ }^{38}$, a 600 Ry real space grid cutoff, DFT-D3 empirical van der Waals corrections ${ }^{39}$, double-zeta plus polarization basis set optimized in molecules $^{40}$ were used in all calculations. A quantum dot of $\sim 1 \mathrm{~nm}$ in diameter in a (32 $\AA)^{3}$ unit cell was prepared starting from bulk $\mathrm{PbS}$ geometry, terminated by $\mathrm{Pb}$ and capped by ligands in the amount to exactly charge-balance the excess of cations. Four chlorobenzene molecules were inserted into available empty spaces in the ligand shell with $\mathrm{Cl}$ oriented both toward and away from the QD and then fully relaxed. Solvent interaction energy was calculated as an average over these four molecules.

Optical and morphological characterization. Steady-state and time-resolved PL measurements were carried out using a Horiba Fluorolog time-correlated singlephoton counting setup, equipped with a photomultiplier tube detector. A monochromatized Xe lamp $\left(\lambda_{\mathrm{ex}}=650 \mathrm{~nm}\right)$ and a pulsed diode laser $\left(\lambda_{\mathrm{ex}}=723 \mathrm{~nm}\right)$ were used as excitation sources for steady-state and time-resolved PL, respectively. Optical absorption measurements were performed using a PerkinElmer Lambda 950 system. The film morphology was characterized using an Asylum Research Cypher AFM and a Hitachi SEM (SU8230).

UPS, XPS, and FTIR measurements. UPS measurement was carried out using a helium discharge source (HeI $\alpha, h v=21.22 \mathrm{eV}$ ). UPS spectra were obtained at a $-5-\mathrm{V}$ bias relative to the spectrometer to efficiently collect low kinetic-energy electrons. XPS measurement was performed using a spectrometer (Thermo Scientific) and a monochromatic K-Alpha X-ray system, with a 75-eV pass energy, and an energy steps of $0.05 \mathrm{eV}$. FTIR spectra were observed using a spectrometer (Thermo Scientific iS50) in a spectral range of $400-4000 \mathrm{~cm}^{-1}$ with ATR accessory.

Reporting summary. Further information on research design is available in the Nature Research Reporting Summary linked to this article.

\section{Data availability}

The data that support the findings of this study are available from the corresponding authors upon reasonable request.

Received: 2 June 2020; Accepted: 1 September 2020;

Published online: 23 September 2020

\section{References}

1. Chuang, C.-H. M., Brown, P. R., Bulović, V. \& Bawendi, M. G. Improved performance and stability in quantum dot solar cells through band alignment engineering. Nat. Mater. 13, 796-801 (2014).
2. Luther J. M. et al. Schottky solar cells based on colloidal nanocrystal films. Nano Lett. 8, 3488-3492 (2008).

3. Choi M.-J. et al. Cascade surface modification of colloidal quantum dot inks enables efficient bulk homojunction photovoltaics. Nat. Commun. 11, 103 (2020).

4. Lee, J.-S., Kovalenko, M. V., Huang, J., Chung, D. S. \& Talapin, D. V. Bandlike transport, high electron mobility and high photoconductivity in allinorganic nanocrystal arrays. Nat. Nanotech. 6, 348-352 (2011).

5. Clifford J. P. et al. Fast, sensitive and spectrally tuneable colloidal-quantumdot photodetectors. Nat. Nanotech. 4, 40-44 (2009).

6. Nikitskiy I. et al. Integrating an electrically active colloidal quantum dot photodiode with a graphene phototransistor. Nat. Commun. 7, 11954 (2016)

7. Won Y.-H. et al. Highly efficient and stable $\mathrm{InP} / \mathrm{ZnSe} / \mathrm{ZnS}$ quantum dot lightemitting diodes. Nature 575, 634-638 (2019).

8. Li Y. et al. Stoichiometry-controlled InP-based quantum dots: synthesis, photoluminescence, and electroluminescence. J. Am. Chem. Soc. 141, 6448-6452 (2019).

9. Sun Q. et al. Bright, multicoloured light-emitting diodes based on quantum dots. Nat. Photon. 1, 717-722 (2007).

10. Lim, J., Park, Y.-S. \& Klimov, V. I. Optical gain in colloidal quantum dots achieved with direct-current electrical pumping. Nat. Mater. 17, 42-49 (2018)

11. Park, Y.-S., Bae, W. K., Baker, T., Lim, J. \& Klimov, V. I. Effect of Auger recombination on lasing in heterostructured quantum dots with engineered core/shell interfaces. Nano Lett. 15, 7319-7328 (2015).

12. Tang, X., Ackerman, M. M., Chen, M. \& Guyot-Sionnest, P. Dual-band infrared imaging using stacked colloidal quantum dot photodiodes. Nat. Photon. 13, 277-282 (2019).

13. Kim J. et al. Butylamine-catalyzed synthesis of nanocrystal inks enables efficient infrared CQD solar cells. Adv. Mater. 30, 1803830 (2018).

14. Chen B. et al. Enhanced optical path and electron diffusion length enable high-efficiency perovskite tandems. Nat. Commun. 11, 1257 (2020).

15. Liu M. et al. Double-sided junctions enable high-performance colloidalquantum-dot photovoltaics. Adv. Mater. 28, 4142-4148 (2016).

16. Azmi, R., Oh, S.-H. \& Jang, S.-Y. High-efficiency colloidal quantum dot photovoltaic devices using chemically modified heterojunctions. ACS Energy Lett. 1, 100-106 (2016).

17. Lu K. et al. High-efficiency PbS quantum-dot solar cells with greatly simplified fabrication processing via "solvent-curing". Adv. Mater. 30, 1707572 (2018).

18. Azmi R. et al. Low-temperature-processed $9 \%$ colloidal quantum dot photovoltaic devices through interfacial management of $\mathrm{p}-\mathrm{n}$ heterojunction. Adv. Energy Mater. 6, 1502146 (2016).

19. Giansante $\mathrm{C}$. et al. Colloidal arenethiolate-capped $\mathrm{PbS}$ quantum dots: optoelectronic properties, self-assembly, and application in solution-cast photovoltaics. J. Phys. Chem. C. 117, 13305-13317 (2013).

20. Aqoma, H. \& Jang, S.-Y. Solid-state-ligand-exchange free quantum dot inkbased solar cells with an efficiency of 10.9\%. Energy Environ. Sci. 11, 1603-1609 (2018).

21. Luther J. M. et al. Structural, optical, and electrical properties of self-assembled films of PbSe nanocrystals treated with 1,2-ethanedithiol. ACS Nano 2, 271-280 (2008).

22. Erslev P. T. et al. Sharp exponential band tails in highly disordered lead sulfide quantum dot arrays. Phys. Rev. B 86, 155313 (2012).

23. You, R. H., Park, Y. J., Lee, H. D., Kim, Y. \& Choi, J. Recent research progress in surface ligand exchange of $\mathrm{PbS}$ quantum dots for solar cell application. Appl. Sci. 10, 975 (2020).

24. Kovalenko, M. V., Scheele, M. \& Talapin, D. V. Colloidal nanocrystals with molecular metal chalcogenide surface ligands. Science 324, 1417-1420 (2009).

25. Aqoma H. et al. High-efficiency photovoltaic devices using trap-controlled quantum-dot ink prepared via phase-transfer exchange. Adv. Mater. 29, 1605756 (2017).

26. Liu M. et al. Hybrid organic-inorganic inks flatten the energy landscape in colloidal quantum dot solids. Nat. Mater. 16, 258-263 (2017).

27. Kim Y. et al. A Facet-specific quantum dot passivation strategy for colloid management and efficient infrared photovoltaics. Adv. Mater. 31, 1805580 (2019).

28. Fischer A. et al. Directly deposited quantum dot solids using a colloidally stable nanoparticle ink. Adv. Mater. 25, 5742-5749 (2013).

29. Kroupa D. M. et al. Tuning colloidal quantum dot band edge positions through solution-phase surface chemistry modification. Nat. Commun. 8, 15257 (2017)

30. Wu, C., De Visscher, A. \& Gates, I. D. Reactions of hydroxyl radicals with benzoic acid and benzoate. RSC Adv. 7, 35776-35785 (2017).

31. Williams K. J. et al. Strong electronic coupling in two-dimensional assemblies of colloidal PbSe quantum dots. ACS Nano 3, 1532-1538 (2009).

32. Bederak $\mathrm{D}$. et al. Comparing halide ligands in $\mathrm{PbS}$ colloidal quantum dots for field-effect transistors and solar cells. ACS Appl. Nano Mater. 1, 6882-6889 (2018). 
33. Biondi M. et al. A chemically orthogonal hole transport layer for efficient colloidal quantum dot solar cells. Adv. Mater. 32, 1906199 (2020).

34. Jo J. W. et al. Enhanced open-circuit voltage in colloidal quantum dot photovoltaics via reactivity-controlled solution-phase ligand exchange. Adv. Mater. 29, 1703627 (2017).

35. Yuan $M$. et al. Doping control via molecularly engineered surface ligand coordination. Adv. Mater. 25, 5586-5592 (2013).

36. VandeVondele, J., Krack, M., Mohamed, F., Parrinello, M., Chassaing, T. \& Hutter, J. Quickstep: fast and accurate density functional calculations using a mixed Gaussian and plane waves approach. Comput. Phys. Commun. 167, 103-128 (2005).

37. Goedecker, S., Teter, M. \& Hutter, J. Separable dual-space Gaussian pseudopotentials. Phys. Rev. B 54, 1703-1710 (1996).

38. Perdew, J. P., Burke, K. \& Ernzerhof, M. Generalized gradient approximation made simple. Phys. Rev. Lett. 77, 3865-3868 (1996).

39. Grimme, S., Ehrlich, S. \& Goerigk, L. Effect of the damping function in dispersion corrected density functional theory. J. Comput. Chem. 32, 1456-1465 (2011).

40. VandeVondele, J. \& Hutter, J. Gaussian basis sets for accurate calculations on molecular systems in gas and condensed phases. J. Chem. Phys. 127, 114105 (2007).

\section{Acknowledgements}

This work was supported by Ontario Research Fund-Research Excellence program (ORF7 Ministry of Research and Innovation, Ontario Research Fund-Research Excellence Round 7), and by the Natural Sciences and Engineering Research Council (NSERC) of Canada. Computations were performed on the Niagara supercomputer at the SciNet HPC Consortium. SciNet is funded by: the Canada Foundation for Innovation; the Government of Ontario; Ontario Research Fund-Research Excellence; and the University of Toronto. This research was also supported by the National Research Foundation (NRF) of Korea (NRF-2020R1A6A3A03038131). We thank D. Kopilovic, E. Palmiano, L. Levina, and R. Wolowiec for the technical support. The authors acknowledge the financial support from QD Solar Inc.

\section{Author contributions}

S.L. and M-J.C. conceptualized the project and contributed to most experimental work. F.P.G.A., S.H., O.V., and E.H.S. supervised the project. G.S. and O.V. performed DFT calculations. M.B. assisted in HDR EQE and NEP measurements. B.C. carried out the perovskite device fabrications. S-.W.B. performed AFM measurements. A.M.N. performed TPC measurements. M.V. performed UPS measurements. J.W. performed XPS measurements. L.K.S performed FTIR measurements. S.L. and M-.J.C. wrote the original manuscript and all authors contributed toward improving it.

\section{Competing interests}

The authors declare no competing interests.

\section{Additional information}

Supplementary information is available for this paper at https://doi.org/10.1038/s41467020-18655-7.

Correspondence and requests for materials should be addressed to O.V. or E.H.S.

Peer review information Nature Communications thanks the anonymous reviewer(s) for their contribution to the peer review of this work.

Reprints and permission information is available at http://www.nature.com/reprints

Publisher's note Springer Nature remains neutral with regard to jurisdictional claims in published maps and institutional affiliations.

Open Access This article is licensed under a Creative Commons By Attribution 4.0 International License, which permits use, sharing,
adaptation, distribution and reproduction in any medium or format, as long as you give appropriate credit to the original author(s) and the source, provide a link to the Creative Commons license, and indicate if changes were made. The images or other third party material in this article are included in the article's Creative Commons license, unless indicated otherwise in a credit line to the material. If material is not included in the article's Creative Commons license and your intended use is not permitted by statutory regulation or exceeds the permitted use, you will need to obtain permission directly from the copyright holder. To view a copy of this license, visit http://creativecommons.org/ licenses/by/4.0/.

(C) The Author(s) 2020 